\title{
Apolipoprotein A-1 in Umbilical Cord Blood of Newborn Infants: Relation to Gestational Age and High-Density Lipoprotein Cholesterol
}

\author{
C. RICHARD PARKER, JR., STEPHEN J. FORTUNATO, BRUCE R. CARR, JOHN OWEN, \\ GARY D. V. HANKINS, AND JOHN C. HAUTH
}

Department of Obstetrics and Gynecology, University of Alabama at Birmingham, Birmingham, Alabama 35294 and The Department of Obstetrics and Gynecology, University of Texas Health Science Center at Dallas, Dallas, Texas 75235 [S. J. F., B. R. C., G. D. V. H.]

\begin{abstract}
Apolipoprotein A-1 (Apo A-1) is the major protein constituent of high-density lipoprotein (HDL) and Apo A-1 plays an important role in lipid metabolism and may be protective against atherosclerosis in adults. However, little is known about HDL and Apo A-1 in the developing human fetus. Herein we investigated the relationship of Apo A-1 levels in umbilical cord blood at delivery to gestational age and HDL cholesterol. Fetal plasma levels of Apo A-1, which were not correlated with those in maternal plasma, were significantly lower among newborns delivered at 21-26 wk gestation $(52 \pm 4.4 \mathrm{mg} /$ dI, mean \pm SE) than in those delivered at 33-34 wk gestation $(87 \pm 5.8 \mathrm{mg} / \mathrm{dl})$. Thereafter, the mean umbilical cord plasma levels of Apo A-1 remained relatively constant $(101 \mathrm{mg} / \mathrm{dl}$ at 39-40 wk of gestation). We found no significant correlations between Apo A-1 levels and fetal sex, race, or delivery method. At equivalent gestational ages and birth weights, however, Apo A-1 levels in white newborns tended to be lower than those in black infants. The Apo A-1/HDL cholesterol ratio in umbilical cord blood rose progressively from 2.5 (27-28 wk gestation) to 3.8 at term, due largely to increased Apo A-1 levels but little change in the mean HDL cholesterol levels, which ranged from $22-24 \mathrm{mg} / \mathrm{dl}$ at each gestational period. These results are suggestive that fetal plasma Apo A-1 is derived solely from fetal sources and that the rate of production and/or clearance of Apo A-1 is altered during the latter third of human intrauterine development. (Pediatr Res 23: 348$351,1988)$
\end{abstract}

\section{Abbreviations}

Apo A-1, apolipoprotein A-1

HDL, high-density lipoprotein

LDL, low-density lipoprotein

Apo A-1 is the major protein constituent of HDL, which plays a major role in cholesterol metabolism by virtue of its capacity for activating lecithin-cholesterol acyltransferase and transporting cholesterol from extrahepatic tissues (1). Thus, HDL is thought to be of importance in counteracting the atherogenic effects of excess cholesterol deposition from LDL, and high

Received April 29, 1987; accepted December 1, 1987.

Correspondence C. Richard Parker, Jr., Ph.D., Department of Obstetrics and Gynecology, University of Obstetrics and Gynecology, University of Alabama at Birmingham, Birmingham, AL 35294.

Supported by NIH Grant HD 22969. plasma levels of Apo A-1 and/or HDL are now considered to afford some protection against atherosclerosis (2).

Despite the recognized importance of Apo A-1, very little is established concerning the ontogeny and regulation of production of this protein in humans. By virtue of our interest in the milieu of the fetus during normal and abnormal intrauterine development, we sought to define the ontogeny of umbilical cord blood levels of Apo A-1 during the latter half of gestation in newborns of women whose pregnancies were uncomplicated.

\section{MATERIALS AND METHODS}

Mixed (arterial and venous) umbilical cord blood was obtained at delivery from 165 newborns of women having no pregnancy complications other than multiple gestation or premature delivery in some. None of the women was treated with glucocorticosteroids in an effort to accelerate fetal lung maturation. Matched sets of maternal venous, umbilical venous, and umbilical arterial blood samples also were obtained at the time of delivery of 15 women at term (38-40 wk gestation). Gestational age, expressed as wk since the last menstrual period, was established based on menstrual history, obstetrical findings, and the Dubowitz examination of the newborn. Data pertaining to newborn weight, sex, and race as well as delivery method were available on all but eight, nine, 11, and six newborns, respectively. Blood was collected either into tubes containing EDTA as anticoagulant or allowed to clot. Plasma or serum was obtained after centrifugation of tubes and stored frozen at $-20^{\circ} \mathrm{C}$ until assay. Informed consent was obtained from the women who participated in this study. The study was reviewed and approved by the Institutional Review Boards of the University of Texas Health Science Center at Dallas and of the University of Alabama at Birmingham, School of Medicine.

Apo A-1 in nonfractionated serum and plasma was measured by a double-antibody radioimmunoassay method using reagents generously provided by Ventrex Laboratories, Inc. The primary antiserum was a mouse monoclonal anti Apo A-1 antibody, and the second antibody was a goat anti-mouse IgG antibody. The assay standard was calibrated against the IUIS/WHO reference standard (CDC 1883). The within- and between-assay coefficients of variation for the Apo A-1 assay were 3.1 and $4.4 \%$, respectively. Because we had either plasma or serum samples for use herein, but not both in most instances, we sought to determine whether there were any systematic differences in the concentration of Apo A-1 in umbilical serum and plasma. Mixed umbilical cord blood from term newborns was drained into tubes containing EDTA and also into tubes containing no anticoagulant $(n=$ five matched pairs). The resulting plasma and serum samples were assayed for Apo A-1 in the same assay procedure. The 
average difference between the pairs was $2.48 \%$ of the mean level of Apo A- 1 for the paired samples, a value roughly equivalent to the within-assay coefficient of variation for the assay. The concentrations of Apo A-1 in serum $(107 \pm 2.3 \mathrm{mg} / \mathrm{dl}$, mean $\pm \mathrm{SE})$ and in plasma $(106 \pm 2.3 \mathrm{mg} / \mathrm{dl})$ from these five newborns were indistinguishable $(p>0.05$, paired $t$ test). We also tested the stability of Apo A-1 during long-term freezing of samples: the mean Apo A-1 level in fresh ( $<2$ months storage) cord serum and plasma from 23 infants (39-43 wk gestation) was $99.2 \mathrm{mg} /$ $\mathrm{dl}$; that in 11 similar-aged infants whose serum and plasma samples had been stored frozen for 2-9 yr before assay was 99.4 $\mathrm{mg} / \mathrm{dl}$. Thus, for the ontogenetic analyses, Apo A-1 levels determined from both fresh and long-term frozen serum or plasma samples were combined for statistical purposes.

Using serum from a group of newborns $(n=69)$ representative of most of the gestational period of interest, we also quantified the levels of HDL cholesterol. Lipoproteins were fractionated according to a modification of the micromethods of Bronzert and Brewer (3). Briefly stated, 175- $\mu$ l aliquots of serum, which were adjusted to a density of $1.06 \mathrm{~g} / \mathrm{ml}$ by the addition of crystalline $\mathrm{KBr}$, were centrifuged in a Beckman LP-42 $\mathrm{Ti}\left(15^{\circ} \mathrm{C}\right.$, $\left.40,000 \mathrm{rpm}, 200,252 \times g_{\text {av }}\right)$ in a Beckman L8-70 ultracentrifuge for $2.5-5 \mathrm{~h}$. rather than for $2.5 \mathrm{~h}$ in a Beckman airfuge $(100,000$ $\mathrm{rpm} ; 135,000 \times g_{\mathrm{av}}$ as originally described (3). The variation in centrifugation time between $2.5-5 \mathrm{~h}$, which was performed for the sake of convenience, did not cause any significant changes in HDL cholesterol levels obtained in a pool of cord serum. Cholesterol in the infranate fraction (HDL) was measured by use of a Beckman Cholesterol Analyzer 2 in which a microenzymatic method was used (3). The within- and between-assay coefficients of variation for cholesterol quantification were both $<5 \%$. The data were subjected to linear regression analysis, paired $t$ test, Shapiro-Wilk test (to test for normalcy of data distribution), analysis of variance, analysis of covariance, and Tukey's Studentized range test by use of Statistical Analysis System.

\section{RESULTS}

Relation between maternal venous, umbilical arterial, and umbilical venous serum levels of Apo $A$ - 1 . At term there was a highly significant and positive relation $(r=0.848, p<0.001)$ between levels of Apo A-1 in umbilical arterial and umbilical venous sera (Fig. 1). The levels of Apo A-1 in umbilical arterial serum $(106 \pm 1.1 \mathrm{mg} / \mathrm{dl}$, mean $\pm \mathrm{SE})$ were slightly higher,
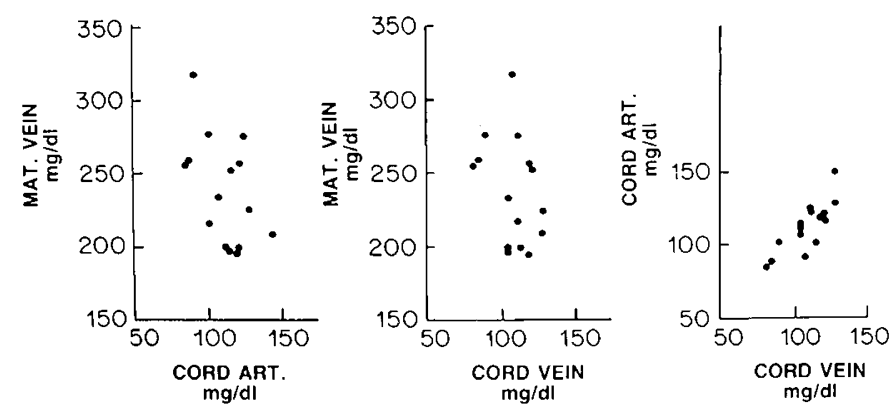

Fig. 1. Relationship among Apo A-1 levels in matched sets of maternal venous, umbilical venous, and umbilical arterial plasma at term. The data for 15 pregnant women and their newborns are plotted individually. although not significantly so ( $p=0.33$, paired $t$ test), than in matched umbilical venous serum $(104 \pm 1.0 \mathrm{mg} / \mathrm{dl})$. However, we found no correlation between Apo A-1 levels in maternal venous serum and those in either umbilical arterial or umbilical venous serum (Fig. 1).

Ontogeny of Apo A-1 in mixed umbilical cord blood. When grouped as a function of gestational age at delivery of the newborn infants, the levels of Apo A-1 in umbilical cord blood varied significantly during the interval of $21-43 \mathrm{wk}$ gestation $(F$ $=4.63, p<0.001)$. There was a progressive increase in the levels of Apo A-1 in mixed umbilical cord blood of newborns delivered between 21 and 34 wk gestation; among newborns delivered thereafter, there was little change as a function of gestational age at delivery in umbilical cord blood levels of Apo A-1 (Table 1). The concentrations of Apo A-1 in mixed umbilical cord blood of newborns delivered at $39-40 \mathrm{wk}(101 \pm 5.3 \mathrm{mg} / \mathrm{dl})$ and at $41-$ $43 \mathrm{wk}(95 \pm 7.0 \mathrm{mg} / \mathrm{dl})$ were significantly higher $(p<0.05)$ than those in newborns delivered at $21-26$ wk gestation $(52 \pm 4.4 \mathrm{mg} /$ dl). Apo A-1 levels among infants delivered at 35-36 and 37-38 wk gestation were somewhat lower than those at 33-34 and at $39-43$ wk gestation. During the interval of 21-34 wk gestation, there was a weak but significant positive correlation between Apo A-1 levels and gestational age $(r=0.143, p=0.002)$ and between Apo A-1 levels and newborn weight $(r=0.144, p=$ 0.003 ). Among newborns delivered thereafter there was no correlation between Apo A-1 levels and gestational age or newborn weight.

Relation of Apo A-1 to sex, race, and delivery method. There was no significant difference between the mean umbilical cord levels of Apo A-1 in 46 male $(88.1 \pm 4.9 \mathrm{mg} / \mathrm{dl}, 2706 \mathrm{~g}$ mean body weight) and 49 female $(87.5 \pm 4.1 \mathrm{mg} / \mathrm{dl}, 2748 \mathrm{~g}$ mean body weight) infants delivered during the interval of 33-43 wk gestation. Among newborns delivered during this same interval, the mean Apo A-1 level in 68 newborns delivered vaginally $(90.4$ $\pm 3.4 \mathrm{mg} / \mathrm{dl}, 2616 \mathrm{~g}$ mean body weight) were slightly, although not statistically, higher than that in 26 newborns delivered by cesarean section $(78.3 \pm 5.5 \mathrm{mg} / \mathrm{dl}, 2753 \mathrm{~g}$ mean body weight). There were too few newborns delivered by cesarean section before the onset of labor to determine whether the presence of labor had any statistically significant effect on Apo A-1 concentrations. We also subdivided the data for Apo A-1 levels as a function of race (Table 2). Within each gestational period, the mean birth weights and gestational ages of black, white, and Latin-American infants were virtually identical. Umbilical cord serum levels of Apo A-1 in white infants were consistently lower than those in black infants; the differences were, however, not statistically significant at any gestational period. Moreover, when analysis of covariance was performed in order to adjust for both gestational age and birth weight, we found no differences in Apo A-1 levels as a function of race. As appeared to be the case from the data in Table 1, there was a slight reduction in Apo A-1 levels in each racial group when delivery occurred at 35-38 wk gestation compared to those at $31-34$ and $39-43$ wk gestation. This reduction does not appear to be attributed to any major differences in the frequency of cesarean section deliveries among the three time periods: $35-38$ wk $=28.9 \%$ cesarean section deliveries; $31-34 \mathrm{wk}=23.3 \%$; and $39-43 \mathrm{wk}=26.5 \%$ cesarean section deliveries.

Relation of Apo A-1 to HDL cholesterol. The ratio of Apo A-

Table 1. Umbilical cord blood levels of Apo A-1 $(\mathrm{mg} / \mathrm{dl})$ as function of gestational age*

\begin{tabular}{|c|c|c|c|c|c|c|c|c|c|}
\hline & \multicolumn{9}{|c|}{ Gestational age (wk) } \\
\hline & $21-26$ & $27-28$ & $29-30$ & $31-32$ & $33-34$ & $35-36$ & $37-38$ & $39-40$ & $41-43$ \\
\hline Mean & $51.9 \dagger$ & $57.6 \dagger$ & $63.8 \dagger$ & $64.9 \dagger$ & 86.9 & 76.9 & 72.0 & 101.4 & 95.2 \\
\hline $\mathrm{SD}$ & 13.8 & 28.1 & 24.8 & 21.4 & 29.3 & 25.7 & 25.6 & 24.9 & 24.2 \\
\hline$n$ & 10 & 16 & 17 & 24 & 26 & 17 & 21 & 22 & 12 \\
\hline
\end{tabular}

* Data from serum and plasma samples were combined for this analysis.

$\dagger$ Significantly lower $(p<0.05)$ than Apo A-1 levels at 33-34 and 39-43 wk of gestation. 
Table 2. Umbilical cord serum levels of Apo A-1 as function of race*

\begin{tabular}{|c|c|c|c|c|c|c|c|c|c|c|c|c|c|}
\hline & & \multicolumn{12}{|c|}{ Gestational age (wk) } \\
\hline & & \multicolumn{3}{|c|}{$21-30$} & \multicolumn{3}{|c|}{$31-34$} & \multicolumn{3}{|c|}{$35-38$} & \multicolumn{3}{|c|}{$39-43$} \\
\hline & & B & W & L & B & W & L & $\mathrm{B}$ & $\mathrm{W}$ & $\mathrm{L}$ & $\mathrm{B}$ & W & L \\
\hline$n$ & & 19 & 19 & 2 & 23 & 18 & 6 & 20 & 11 & 2 & 12 & 5 & 14 \\
\hline \multirow{2}{*}{$\begin{array}{l}\text { Apo A-1 (mg/ } \\
\text { dl) }\end{array}$} & Mean & 60.7 & 53.0 & 73.5 & 83.4 & 76.9 & 58.3 & 81.8 & 70.3 & 52.0 & 102 & 88.6 & 100 \\
\hline & $\mathrm{SD}$ & 21.9 & 24.7 & 36.1 & 31.7 & 21.9 & 20.0 & 25.7 & 20.4 & 39.6 & 27.4 & 21.4 & 25.8 \\
\hline \multirow{2}{*}{$\begin{array}{c}\text { Gestational } \\
\text { age (wk) }\end{array}$} & Mean & 27.5 & 27.8 & 29.8 & 32.6 & 32.8 & 32.5 & 36.6 & 36.4 & 37.0 & 40.5 & 40.1 & 40.4 \\
\hline & SD & 2.3 & 1.4 & 0.4 & 1.1 & 1.1 & 0.8 & 1.2 & 1.2 & 1.4 & 1.3 & 1.1 & 1.4 \\
\hline \multirow[t]{2}{*}{ Birth wt $\dagger(g)$} & Mean & 999 & 1038 & 1183 & 1897 & 1872 & 1732 & 2406 & 2410 & 2163 & 3310 & 3545 & 3406 \\
\hline & SD & 293 & 301 & 159 & 354 & 243 & 442 & 554 & 583 & 293 & 572 & 556 & 467 \\
\hline
\end{tabular}

* B, black; W, white; L, Latin American.

$\dagger$ Birth weights were missing on six newborns for whom Apo A-1 levels are presented.

$1(\mathrm{mg} / \mathrm{dl})$ to HDL cholesterol $(\mathrm{mg} / \mathrm{dl})$ in mixed umbilical cord serum (or plasma) was determined in 69 newborn infants (Fig. 2). This ratio rose during gestation from 2.5 at $27-28$ wk gestation to 3.8 at term. There were, however, no statistically significant ( $p>0.05$, analysis of variation) variations in this ratio among the various age groups of newborns. The change in the Apo A-1 to $\mathrm{HDL}$ cholesterol ratio in plasma was attributable to a rise in Apo A-1 levels with virtually no change in HDL cholesterol levels during the gestational interval that we examined; the mean HDL cholesterol level of each gestational age group varied from $22-24 \mathrm{mg} / \mathrm{dl}$.

\section{DISCUSSION}

The physiological role of HDL in lipid transport as well as the capacity for high levels of HDL and Apo A-1 to offer some protection against atherosclerosis in adulthood are recognized (1, 2). It also is reasonably well established that the profile of lipoproteins seen in adults are established early in childhood (4, 5 ). Whereas the plasma levels of HDL lipids and apolipoproteins during various circumstances of postnatal life have been described, there is little information regarding the regulation of production and the ontogenetic changes in HDL in the developing human fetus.

In our cross-sectional analysis, we and others (6-9) found that fetal plasma levels of Apo A-1 are substantially lower at term than are those in adults $(2,5,7,8)$. We found a progressive rise in Apo A-1 levels in umbilical cord blood at the time of delivery over the interval of 21-34 wk gestation. Thereafter, fetal plasma levels of Apo A-1 remained relatively constant through term. These results are qualitatively similar to those of Ginsburg and Zetterstrom (10) and of Davidsen (11), who found, in a much smaller series of infants, that maximal HDL (protein) levels were achieved by about 33 wk gestation. We found no correlation between Apo A-1 levels and fetal sex or mode of delivery. At equivalent gestational periods and newborn weights, umbilical cord levels of Apo A-1 consistently tended to be higher, although not statistically, in black infants than in white infants. The ratio of Apo A-1 to HDL cholesterol in fetal plasma at term (3.8) is slightly higher than are values that can be computed for normal adults from the data of Naito (3.2) (2) and of Freedman et al. (2.3) (5). Our data for the Apo A-1/HDL ratio during gestation, although not showing statistically significant changes, is nevertheless suggestive of a progressive rise in the protein to lipid ratio in HDL particles during the third trimester. Such a change is perhaps to be expected because we also have found that there is a progressive decline of the total and LDL cholesterol levels in fetal plasma during the last 8-12 wk gestation (12) that appears to be caused by a progressive increase in the rate of assimilation of LDL cholesterol by the fetal adrenals for use in steroidogenesis $(12,13)$. Thus, there likely would be progressively less cholesterol deposited in other tissues that would be available for incorporation into HDL during the same gestational interval.

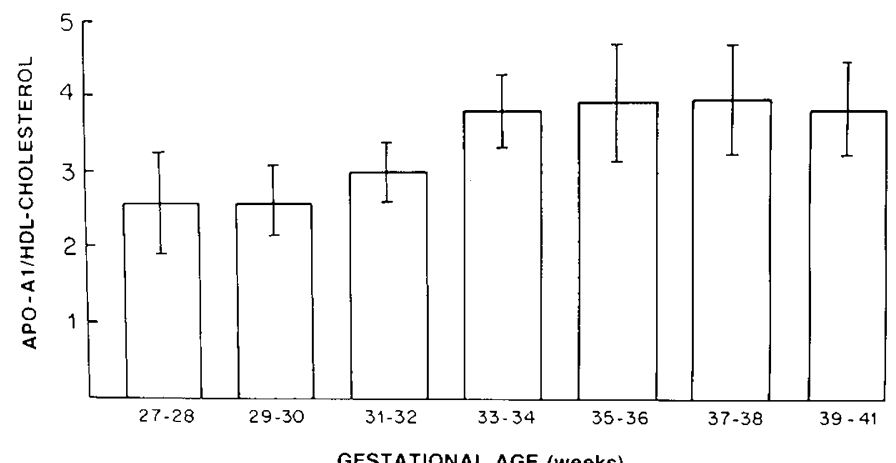

Fig. 2. Relationship of Apo A-1 to HDL cholesterol levels in umbilical cord plasma during gestation. The ratio of Apo A-1 (mg/dl) to HDL cholesterol $(\mathrm{mg} / \mathrm{dl})$ in plasma of each newborn was determined. These data are are presented as the mean $\pm \mathrm{SE}$ at each gestational period.

The mechanisms responsible for the progressive rise in umbilical cord plasma levels of Apo A-1 during the interval of 21-33 wk, with no further substantial increases thereafter, are unclear at the present. We found no evidence for a maternal or placental contribution to fetal plasma Apo A-1 levels (i.e. no relationship between maternal and fetal plasma levels or of a gradient between umbilical venous and umbilical arterial levels of Apo A-1). Therefore, the ontogenetic changes in fetal plasma levels of Apo A-1 probably come about as changes in fetal homeostasis that result from either altered rates of synthesis in fetal intestine and liver (14) or of clearance in the fetal liver. Sex steroids, and especially estrogens, are believed to regulate the synthesis of Apo A-1 $(14,15)$. The temporal pattern of change in fetal plasma levels of Apo A-1 and of estrogens are, however, different. Whereas maximal levels of Apo A-1 occur before term, umbilical cord serum levels of estriol rise progressively from 23-40 wk of gestation (16), and those of estradiol appear to remain relatively constant throughout the third trimester (17). Moreover, we find that in anencephalic newborns, who have deficiencies in estrogens and several other steroid hormones $(13,18)$, but who are hypercholesterolemic (13), plasma Apo A-1 levels are generally within the upper limits of the normal range (19). Exposure of the fetus to synthetic glucocorticosteroids has been shown to increase HDL cholesterol $(19,20)$ as well as Apo A-1 levels (19, 20 ). However, there is no correlation between the time course of changes in fetal plasma levels of Apo A-1 and those of endogenous glucocorticosteroids during development (21). Thus, the factors that regulate production (as well as plasma clearance) of Apo A-1 in the fetus remain to be defined.

Acknowledgments. The authors thank Ventrex Laboratories, Inc., for their generous gift of the reagents for Apo A-1 radioimmunoassay. We also thank Sholeh Golkar and Kim Rivera for technical assistance, Barbara Quinnelly for editorial help, and 
David Guzick, M.D., Ph.D. for suggestions in our statistical analyses.

\section{REFERENCES}

1. Havel RJ 1980 Lipoprotein biosynthesis and metabolism. Ann NY Acad Sci 348:16-27

2. Naito HK 1985 The association of serum lipids, lipoproteins, and apolipoproteins with coronary artery disease assessed by coronary arteriography. Ann NY Acad Sci 454:230-238

3. Bronzert TJ, Brewer HB Jr 1977 New Micromethod for measuring cholesterol in plasma lipoprotein fractions. Clin Chem 23:2089-2098

4. Mellies MJ, Laskarewski PM, Trent T, Glueck CJ 1985 Tracking of high- and low-density-lipoprotein cholesterol from childhood to young adulthood in a single large kindred with familial hypercholesterolemia. Metabolism 34:747753

5. Freedman DS, Srinivasan SR, Shear CL, Franklin FA, Webber LS, Berenson GS 1986 The relation of Apolipoproteins A-1 and B in children to parental myocardial infarction. N Engl J Med 315:721-726

6. Dolphin PJ, Breckenridge WC, Dolphin MA, Tan MH 1984 The lipoproteins of human umbilical cord blood. Apolipoprotein and lipid levels. Atherosclerosis $51: 109-122$

7. McConathy WJ, Lane DM 1980 Studies on the apolipoproteins and lipoproteins of cord serum. Pediatr Res 14:757-761

8. Pocovi M, Ordovas JM, Grande F 1983 Lecithin: Cholesterol acyltransferase, lipids and lipoproteins in maternal and umbilical cord plasma. Artery 11 264-272

9. Van Biervliet JP, Vercaemst R, DeKeersgieter W, Vinaimont N, Caster H, Rousseneu M 1980 Evolution of lipoprotein patterns in newborns. Acta Paediatr Scand 69:593-596

10. Ginsburg BE, Zetterstrom R 1977 High density lipoprotein concentrations in newborn infants. Acta Paediatr Scand 66:39-41
11. Davidsen O 1974 Immunoelectrophoretic determination of serum globins in newborn infants of diabetic mothers. Acta Paediatr Scand 63:833-838

12. Parker CR Jr, Carr BR, Simpson ER, MacDonald PC 1983 Decline in the concentration of low-density lipoprotein-cholesterol in human fetal plasma near term. Metabolism 32:919-923

13. Parker CR Jr, Carr BR, Winkel CA, Casey ML, Simpson ER, MacDonald PC 1983 Hypercholesterolemia due to elevated low density lipoprotein-cholesterol in newborns with anencephaly and adrenal atrophy. J Clin Endocrinol Metab 57:37-43

14. Zannis VI, Kurnit D, Breslow JL 1982 Hepatic Apo A-1 and Apo-E and intestinal Apo A-1 are synthesized in precursor isoprotein forms by organ cultures of human fetal tissues. J Biol Chem 257:536-544

15. Archer TK, Tam SP, Deeley RG 1986 Kinetics of estrogen-dependent modulation of apolipoprotein A-1 synthesis in human hepatoma cells. J Biol Chem 261:5067-5074

16. Parker CR Jr, Hankins GDV, Guzick DS, Rosenfeld CR, MacDonald PC 1987 Ontogeny of unconjugated estriol in fetal blood and the relation of estriol levels at birth to the development of respiratory distress syndrome. Pediatr Res 21:386-390

17. Ho Yuen B, Phillips WDP, Cannon W, Sy L, Redford D, Burch P 1982 Prolactin, estradiol, and thyroid hormones in umbilical cord blood of neonates with and without hyaline membrane disease: a study of 405 neonates from mid-pregnancy to term. Am J Obstet Gynecol 142:698-703

18. Parker CR Jr, Leveno KJ, Milewich L, MacDonald PC 1986 Lecithin-sphingomyelin ratios in amniotic fluid of pregnancies with an anencephalic fetus. Obstet Gynecol 68:546-548

19. Parker CR Jr, MacDonald PC, Carr BR, Morrison JC 1987 The effects of dexamethasone and anencephaly on newborn serum levels of Apolipoprotein A-1. J Clin Endocrinol Metab 65:1098-1101

20. Andersen GE, Friis-Hansen B 1977 Cord serum lipid and lipoprotein-cholesterol values in normal and betamethasone-treated newborns of varying gestational age. Acta Paediatr Scand 66:355-360

21. Hauth JC, Parker CR Jr, MacDonald PC, Porter JC, Johnston JM 1978 A role of fetal prolactin in lung maturation. Obstet Gynecol 51:81-88 